\title{
Design Challenges for a Wide-Aperture Insertion Quadrupole Magnet
}

\author{
S. Russenschuck, B. Auchmann, J. C. Perez, D. Ramos, P. Fessia, M. Karppinen, G. Kirby, T. Sahner, and \\ N. Schwerg
}

\begin{abstract}
The design and development of a superconducting (Nb-Ti) quadrupole with 120-mm aperture, for an upgrade of the LHC insertion region, faces challenges arising from the LHC beam optics requirements and the heat-deposition. The first triggered extensive studies of coil alternatives with four and six coil-blocks in view of field quality and operation margins. The latter requires more porous insulation schemes for both the cables and the ground-plane. This in turn necessitates extensive heat propagation and quench-velocity studies, as well as more efficient quench heaters. The engineering design of the magnet includes innovative features such as self-locking collars, which will enable the collaring to be performed with the coils on a horizontal assembly bench, a spring-loaded and collapsible assembly mandrel, tuning-shims for field quality, porous collaring-shoes, and coil end-spacer design based on differential geometry methods. The project also initiated code extensions in the quench-simulation and CAD/CAM modules of the CERN field computation program ROXIE.
\end{abstract}

Index Terms-Low-beta insertions, magnet design, superconducting quadrupole.

\section{INTRODUCTION}

$\mathbf{T}$ HE DESIGN and construction of a wide-aperture, superconducting quadrupole magnet for the LHC insertion region, hereafter denoted $\mathrm{MQXC}$, is part of a study towards a luminosity upgrade of the Large Hadron Collider at CERN [1]-[3]. The effort is part of the SLHC-PP project, which includes funding from the European Union in the PF7 framework. The design and development work presented in this paper is constrained by the use of existing superconducting cables (inner and outer layer cables of the LHC main dipole), and the in-house production of the models and series quadrupole magnets using tooling recovered from the LHC dipole magnet production. We will review the technical challenges of the MQXC, which led to design features such as more porous cable insulation [4], [5], a new collar structure allowing horizontal assembly with a hydraulic collaring press, tuning shims [6] for the adjustment of field quality, a fishbone like structure for the ground-plane insulation, and an improved quench-heater design. The production will have to follow established procedures for the curing and assembly of the coils in order to match the workflow established in CERN's "large magnet facility." The

Manuscript received August 02, 2010; accepted December 29, 2010. Date of publication February 22, 2011; date of current version May 27, 2011.

The authors are with CERN, CH-1211 Geneva 23, Switzerland (e-mail: stephan.russenschuck@cern.ch).

Color versions of one or more of the figures in this paper are available online at http://ieeexplore.ieee.org.

Digital Object Identifier 10.1109/TASC.2011.2105453

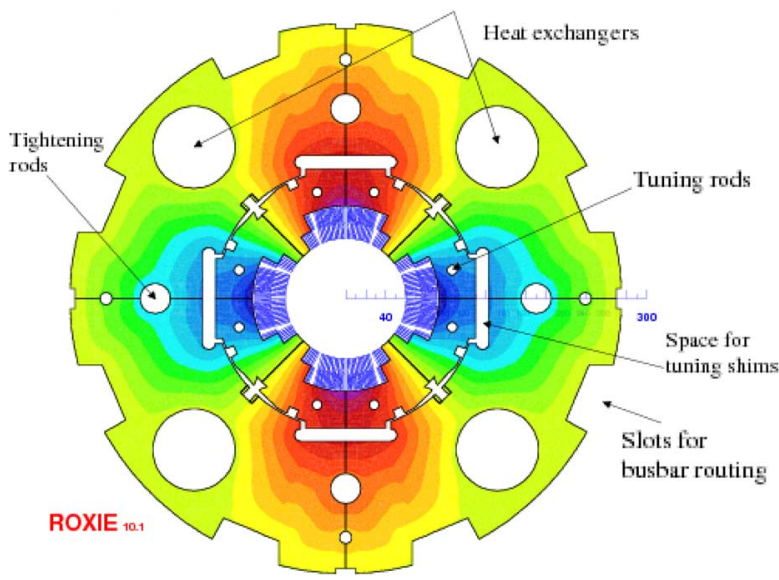

Fig. 1. Coil, collar, and yoke cross sections with iso-surfaces of the magnetic vector potential $A_{z}$ (field lines).

design process takes full advantage of the recently improved software tools [7], featuring quench simulation, 3D coil optimization, and ROXIE-CATIA interfacing for the making of drawings and the manufacturing of coil-end spacers. Extensive heat deposit studies [8] have revealed that the heat-load to the inner triplet magnets, on the order of $500 \mathrm{~W}$, requires two bayonet heat exchangers with minimum inner diameters of $65 \mathrm{~mm}$, to keep the maximum vapor velocity below $7 \mathrm{~ms}^{-1}$ [9]. The iron yoke design can easily accommodate these heat exchangers (and pumping lines) and now allows also for the placing of tuning shims and rods to compensate for multipole field errors. This is particularly important for the small production batch of less than 20 magnets. The cross section of the quadrupole magnet is shown in Fig. 1. Its main parameters are summarized in Table I.

\section{Porous InSUlation SCHEME}

The bath temperature of the coolant is estimated to be $1.96 \mathrm{~K}$ [9], which is at the maximum of the thermal conductivity of superfluid helium. Because the thermal conductivity drops sharply above $1.96 \mathrm{~K}$, the additional temperature gradient to the heat exchanger has to be kept at a minimum. Therefore a more porous insulation scheme for the superconducting cable was proposed [5]. However, this insulation has a considerably lower elastic modulus with respect to the LHC main-magnet insulation scheme. This was shown with 10-stack tests, which also show an offset (with respect to the LHC standard insulation) in the thickness of the cable insulation under the assumed pressure after cooldown to cryogenic temperatures. This is one of the reasons for the construction of an "instrumented collar 
TABLE I

MQXC PARAMETER LIST. OL = Outer Layer, IL = Inner Layer, $I_{\text {nom }}=$ Nominal Current AT $127 \mathrm{Tm}^{-1}$, NONLINEAR CALCULATION (2D), $L=$ Self Inductance, AND $L_{d}=$ Differential Self Inductance

\begin{tabular}{|c|c|c|}
\hline Magnet aperture & 120 & $\mathrm{~mm}$ \\
\hline Magnetic lengths, Q1 / Q2 & $9160 / 7760$ & $\mathrm{~mm}$ \\
\hline Nominal gradient & 127 & $\mathrm{Tm}^{-1}$ \\
\hline$I_{\text {nom }}$ & 13800 & A \\
\hline Max. gradient & 149.7 & $\mathrm{Tm}^{-1}$ \\
\hline$I_{\max }$ & 16340 & A \\
\hline Yoke outer diameter & 550 & $\mathrm{~mm}$ \\
\hline Total axial forces at $I_{\mathrm{nom}}$ & 417 & $\mathrm{kN}$ \\
\hline Work. point on load line IL / OL & $79.9 / 83.2$ & $\%$ \\
\hline Operating temperature & 1.9 & $\mathrm{~K}$ \\
\hline Temperature margin IL / OL & $2.12 / 2.21$ & $\mathrm{~K}$ \\
\hline$L$ at $I_{\text {nom }}$ & 5.05 & $\mathrm{mHm}^{-1}$ \\
\hline$L_{\mathrm{d}}$ at $I_{\mathrm{nom}}$ & 4.78 & $\mathrm{mHm}^{-1}$ \\
\hline Cable dim. IL (insulated) & $15.42,2.002,2.337$ & $\mathrm{~mm}$ \\
\hline Cable dim. OL (insulated) & $15.42,1.649,1.890$ & $\mathrm{~mm}$ \\
\hline $\mathrm{Cu} / \mathrm{SC}$ ratio, IL / OL & $1.65 / 1.95$ & \\
\hline Insulation thickness IL / OL & $0.135 / 0.145$ & $\mathrm{~mm}$ \\
\hline Number of strands IL / OL & $28 / 36$ & $\mathrm{~mm}$ \\
\hline Strand diam., IL / OL & $1.065 / 0.825$ & $\mathrm{~mm}$ \\
\hline
\end{tabular}

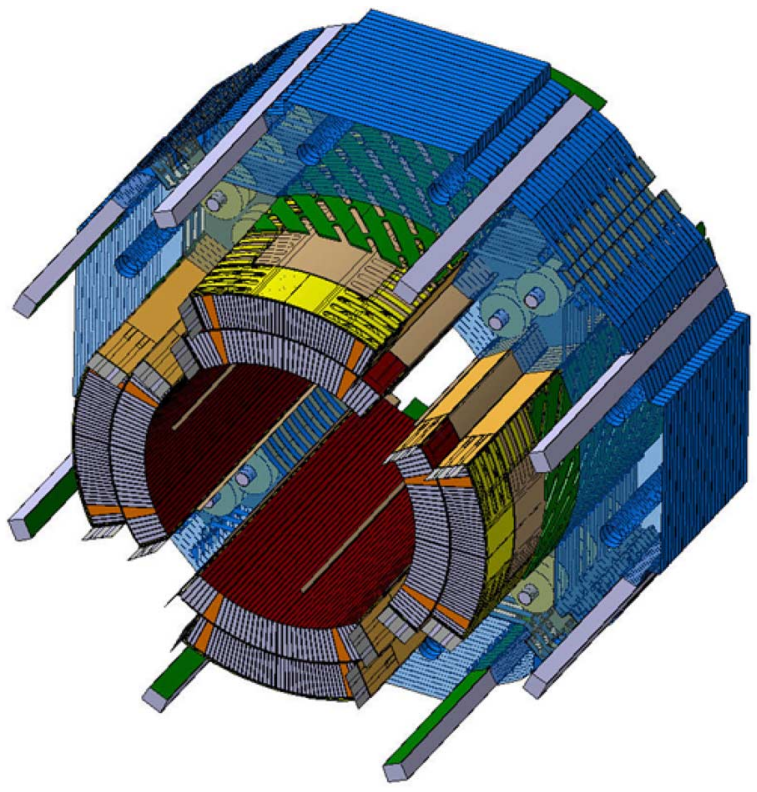

Fig. 2. Instrumented collar pack of $120 \mathrm{~mm}$ long, showing the porous groundplane insulation.

pack", which will allow the measurement of the resulting coil stress under realistic assembly conditions. An artist's view of the collar pack is shown in Fig. 2. The figure also shows the new porous ground plane insulation scheme that maximizes the heat transfer through the collar and yoke laminations to the heat exchanger. A low elastic modulus also results in a considerably larger coil after winding and curing. Thus a sophisticated spring-loaded, collapsible assembly mandrel had to be developed.

\section{Electromagnetic Design}

The magnet cross section and 3D coil design was already performed in 2008 [3]. The coil cross section is shown in Fig. 3 together with the thermal margin (left) and the enthalpy reserve (right). The volumetric enthalpy reserve of the supercon-

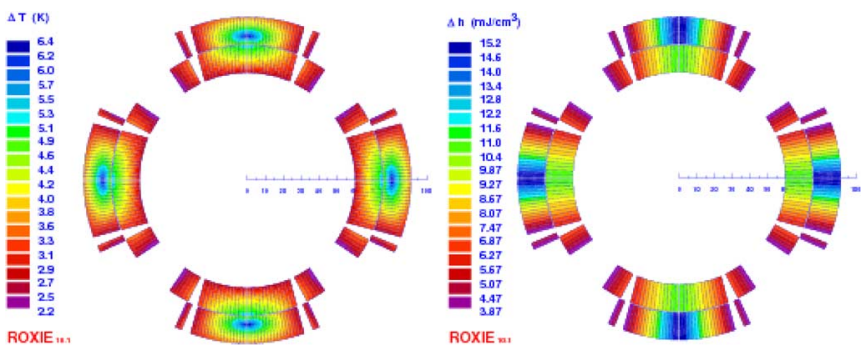

Fig. 3. (Left) Thermal margin. (Right) Volumetric enthalpy reserve. Both at nominal excitation and coil temperature of $1.9 \mathrm{~K}$.

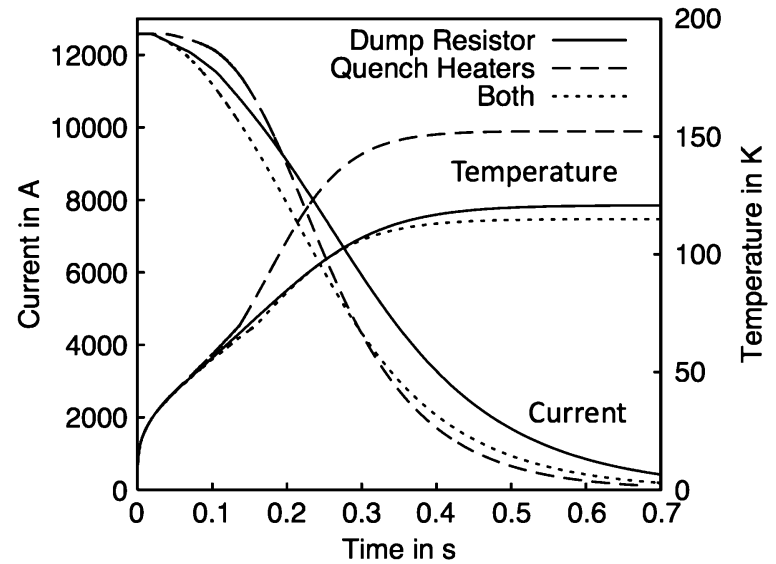

Fig. 4. Current decay and hot-spot temperature after a quench in the MQXC magnet. Quench detection voltage $( \pm 0.1 \mathrm{~V})$. Quench heater Power $(30 \mathrm{~W} / \mathrm{m})$. Heater time constant $(37.7 \mathrm{~ms})$. Heater delay $(40 \mathrm{~ms})$. Extraction resistor (40 $\mathrm{m} \Omega$ ).

ducting wires is defined by $\Delta h:=\int_{T_{\mathrm{b}}}^{T_{\mathrm{c} J, B)}} \rho c_{\mathrm{p}}(T) \mathrm{d}$, where $[h]=1 \mathrm{Jm}^{-3} \cdot T_{\mathrm{b}}$ is the bath temperature of the coolant and $T_{c}(J, B)$ critical temperature as a function of current density and applied magnetic flux density. $\rho c_{\mathrm{p}}$ is the weighed sum of the volumetric heat capacities (VHC) of copper and superconductor in a strand. We will focus in this section on magnet protection issues, the study of an alternative coil cross section and its impact on persistent currents, as well as the coil-end design and endspacer manufacturing.

\section{A. Magnet Protection}

Detailed quench simulation studies have shown that it will be possible to protect the magnet by either the firing of quenchback heaters or the switching-in of an extraction resistor. The use of both protection techniques simultaneously does not yield any reduction of the hot-spot temperature, as in this case the normal zone in the outer layer cable (with less stabilizing copper) leads to higher ohmic losses and thus a higher peak temperature; see Fig. 4.

However, magnet protection with only an extraction resistor (dimensioned such that the terminal voltage at the magnet does not exceed $400 \mathrm{~V}$ ) relies on the quench-back mechanism. Quench-back is a normal zone generation due to induced losses from the interstrand and interfilament coupling currents. The quench-back mechanism depends on empirical data (such as the contact resistances between strands and the residual resistivity ratio RRR) and thus needs to be measured. Although the cable parameters are known from the extensive testing of the LHC 


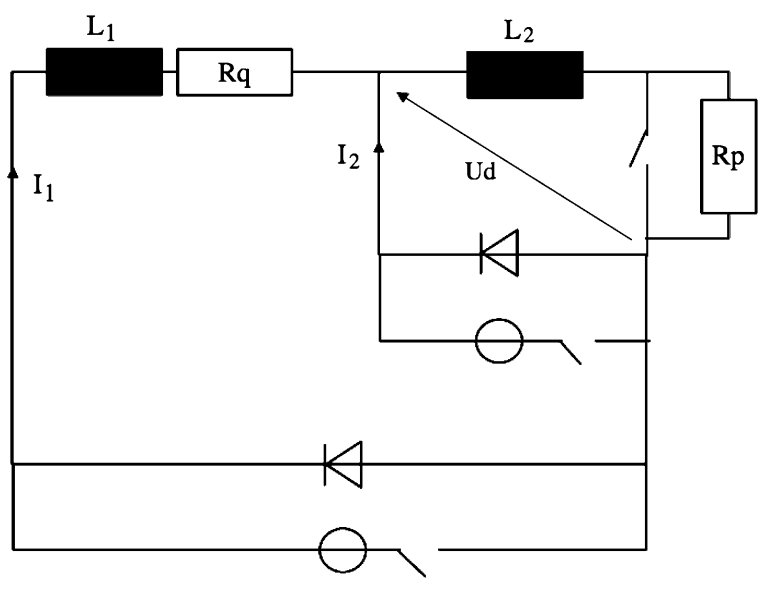

Fig. 5. Nested powering scheme for magnets Q1 and Q2a. If Q1 quenches (inductance $L_{1}$ and normal zone resistance $R_{\mathrm{q}}$ ), although the current in Q2 is higher, the switching-in of the protection resistor is inefficient as long as the free-wheeling diode is conducting.

main dipole magnets, they will be influenced by the enhanced cooling, which also influences the quench heater delay. It has been decided to test a 2-m-long model magnet in a horizontal cryostat on the CERN magnet-test benches using both quench heaters and a dump resistor.

Because of space-constraints in the insertion regions of the LHC, a nested scheme with two power supplies was proposed. If magnet Q1 quenches (inductance $L_{1}$ and normal zone resistance $R_{\mathrm{q}}$ ), although the current in Q2 is higher, the switching-in of the protection resistor is inefficient as long as the free-wheeling diode (forward voltage Ud) is conducting; see Fig. 5. Additional quench simulation studies will be necessary to exclude potentially dangerous quenches in the two nested circuits.

The more porous insulation scheme allows for a factor 4 higher heat extraction. The new open ground insulation introduces about $30 \%$ of open channels for the superfluid helium passage. However, this extra cooling will degrade the standard quench heater performance. As a result, the heater must provide for more heat at the coil, available at a delay time that is shorter than in the LHC main dipoles and quadrupoles.

The heating element is made from $15-\mathrm{mm}$-wide, $0.025-\mathrm{mm}$ thick stainless steel (304) sheets chemically etched to produce a snake-like form and to avoid burrs that could endanger the integrity of the insulation. The heater, shown in Fig. 6, is a sandwich of a $0.075 \mathrm{~mm}$ Kapton sheet, the heating element, another 0.075-mm-thick Kapton sheet, a second heating element, and an outer Kapton layer of again $0.075 \mathrm{~mm}$ thickness. Copper plating of the heater elements is done at their extremities to provide a connection surface for the current leads. The heater is located between the two layers of the coil, in order to cover as many superconducting coil turns as possible.

\section{B. Persistent Currents}

Flux pinning is responsible for magnetic hysteresis and thus for multipole field errors that vary with the powering cycle of the magnets. It is thus impossible to suppress both the geometric field errors and those due to superconducting filament magnetization. Still, some differences between coil designs persist. We have studied two variants of the MQXC quadrupole coil

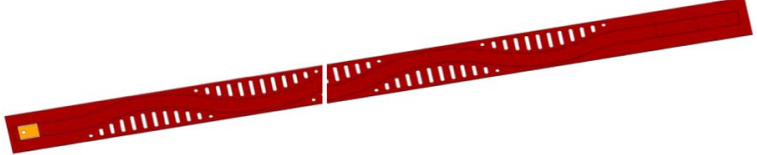

Fig. 6. Sandwich of two quench heaters to be located in between the inner and outer layer coils of MQXC.

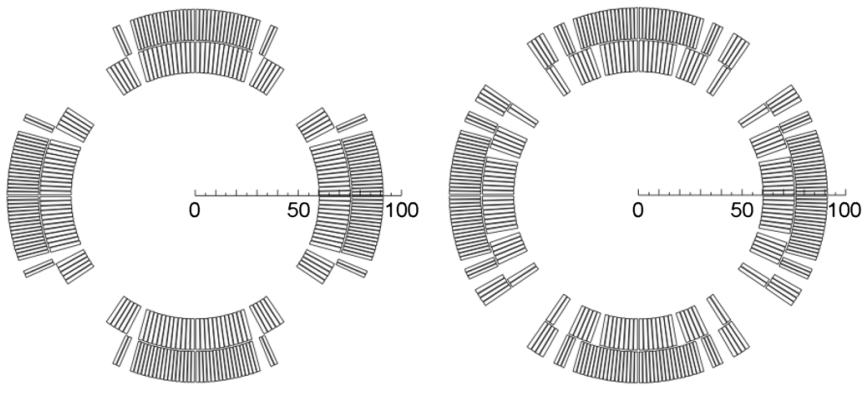

Fig. 7. Two coil cross sections for a 120 -mm-aperture quadrupole, referred to as (left) the 4-block [3] and (right) the 6-block design.

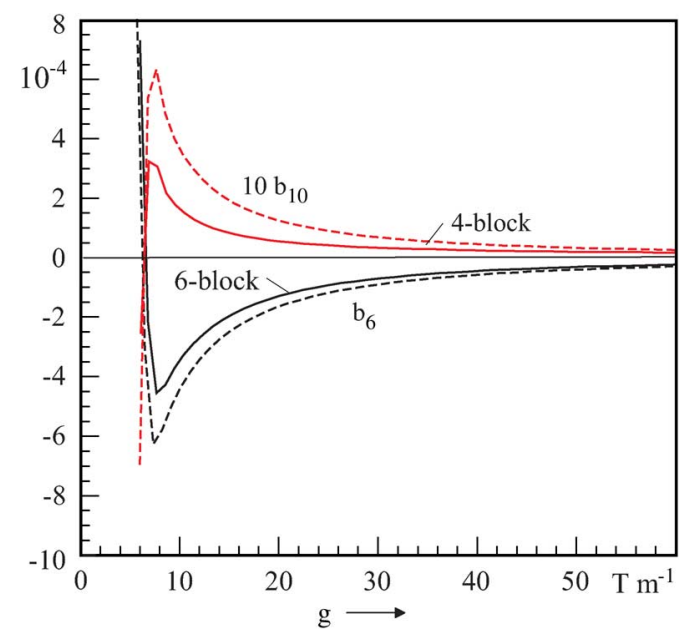

Fig. 8. Multipole field errors b6 and b10 at the reference radius of $40 \mathrm{~mm}$ as a function of the gradient. No iron saturation has been taken into account. The memory sequence is $\left\{0, I_{\text {nom }} / 2,0.05 I_{\text {nom }}, I_{\text {inj }}\right\}$. (Solid line) The 6-block coil and (dashed line) the 4-block coil.

as shown in Fig. 7. The variants are denoted 4-block (left) and 6-block coil (right), respectively. The 6-block coil contains a low current-density block (inner layer cable) in the high-field region of the outer layer, a design feature that was developed for the LHC inner-triplet quadrupoles [10].

The relative multipole errors $b_{6}$ and $b_{10}$ at a reference radius of $40 \mathrm{~mm}$ are given in Fig. 8. The reason for the lower persistent-current-induced field error in the 6-block coil is that both coil layers individually have minimal geometrical field errors. In the 4-block coil, a high dodecapole field error caused by the inner layer coil (128 units in $10^{-4}$ at $40 \mathrm{~mm}$ reference radius) is compensated for by the outer layer. While this works well for the geometrical field errors, persistent current effects do not cancel because of the different field levels and filament sizes in the inner and outer layer cables.

Nevertheless, it was decided to continue with the 4-block design in order to be compatible with the work-flow established in CERN's "large magnet facility", by avoiding the inter layer 


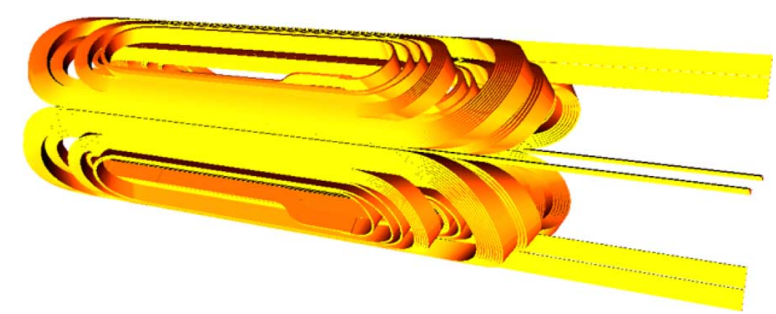

Fig. 9. MQXC coil with lead- and return-ends. The straight section is shortened to $300 \mathrm{~mm}$.

splice and allowing the curing of the inner and outer layer coils separately. Furthermore, the field quality at injection field level is not critical for the machine performance and the effect of manufacturing errors can be compensated for by tuning shims and rods, see Fig. 1. Filling all the foreseen spaces with shims and rods (made from the low-carbon yoke steel) raises the b6 field component by 0.93 and 0.62 units, respectively. Filling only the top/bottom or the left/right spaces results in $b_{4}= \pm 4.1$ units for the shims and \pm 2.2 units for the rods. If required, the rods and shims can also be used to compensate skew field components due to up/down asymmetries: \pm 8.2 units of skew dipole and \pm 1.32 units of skew sextupole.

\section{End-Spacer Design and Manufacture}

The MQXC magnet design takes full advantage of the features implemented in the CERN field computation program ROXIE [7]. In particular, the design and manufacture of the coil end-spacers follows the integrated approach with data transfer from ROXIE to CAD (CATIA) and the 5-axis CNC machining of the pieces. The machined surfaces are developable surfaces, which are described by their base-curves on the winding mandrel and the Darboux vectors to this space curve. The Darboux vectors define the "rulings" along which the surface can be unrolled (developed) into a plane geodesic strip. The coil-end design begins with finding the optimal distribution and spacing of the coil blocks in the ends. A virtual realty preview of the entire coil is shown in Fig. 9.

The shapes of the spacer surfaces are then optimized with the aim of minimizing the cable's deformation energy. For this purpose it is assumed that it will be much more difficult to bend the cable across its narrow face than it is to bend it across its broad face. The two bending directions are thus often referred to as the "hard way" and the "easy way". Because of the torsion, stemming from the cable's position in the magnet cross section, it is not possible to avoid any hard-way bend. Its minimization is therefore done in an iterative way.

A newly developed interface to CATIA allows the transfer of data to the manufacturer in an industrial standard such as STEP. The main challenge for the programming of this interface was to avoid a default interpolation of the vertex points on the cable's base-curve and free edge by the CAD program, as this would result in a loss of information on the Darboux vectors.

Winding tests have shown that the best results are obtained if the left-lay cables are wound in counter-clockwise direction. The internal cable joint requires that the two coil layers be wound in opposite direction. Therefore, the less stable outer

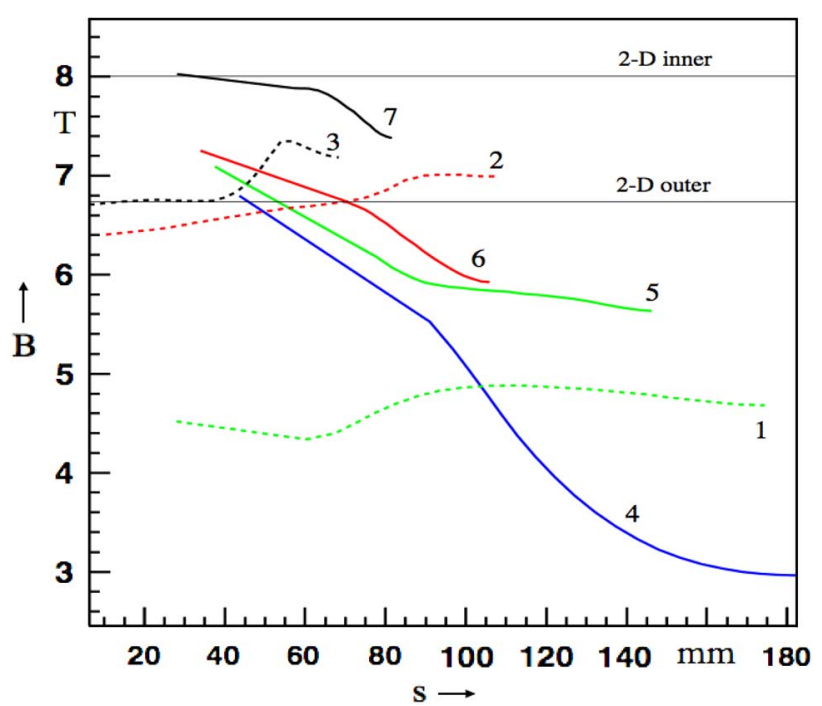

Fig. 10. Peak fields in the coil blocks of the non-connection (return) end. The arc lengths of the conductors is measured from the onset of the coil end towards the nose.

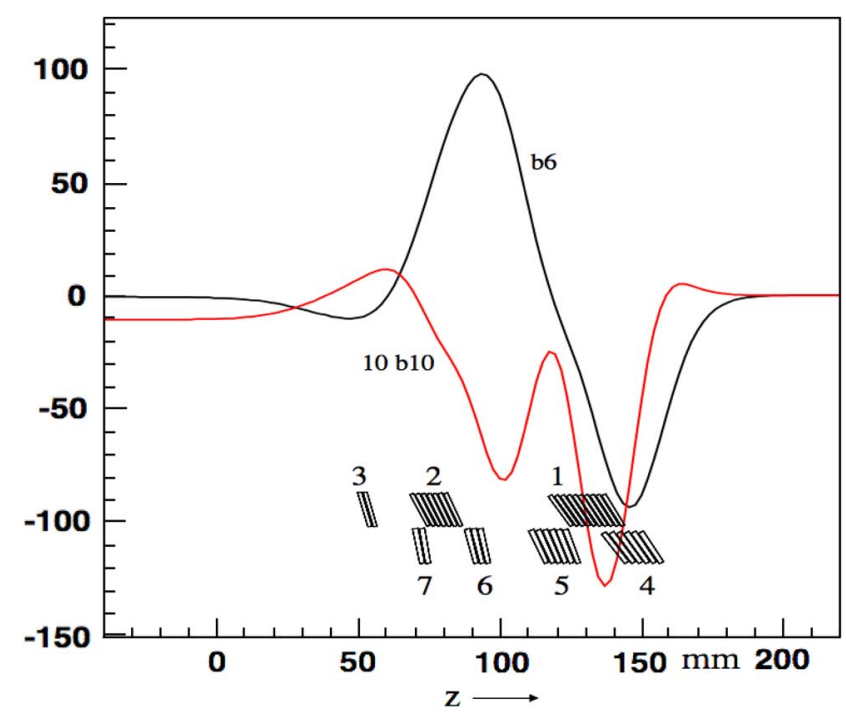

Fig. 11. Variation of the $b_{6}$ and $b_{10}$ field component as a function of the longitudinal position $(z)$ in the return end.

layer cable is wound counter-clockwise, while the inner-layer cable is wound clockwise.

\section{Field Optimization in the Coil Ends}

The relative position of the blocks in the coil ends of the magnet must be chosen in order to minimize the integrated field components and the peak field enhancement. Fig. 10 shows the modulus of the magnetic flux density as a function of the arc length in the coil end (from the straight section toward the nose). The blocks No. 2 and 3 in the outer layer show a local peak field enhancement near the nose. This corresponds to $83.2 \%$ on the load line and is thus the limiting factor for the magnet performance. The inner layer blocks show no local peak field enhancement in the coil ends. The field enhancement is lower at the non-connection side compared to the return end.

Fig. 11 shows the field quality ( $b_{6}$ and $b_{10}$ components at 40 $\mathrm{mm}$ reference radius) as a function of the longitudinal position. 


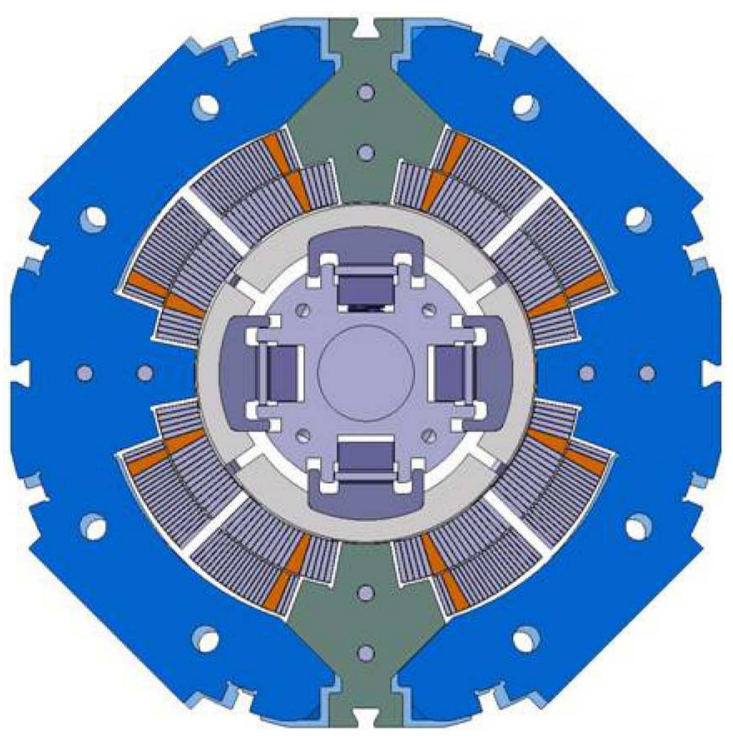

Fig. 12. Rendering of the spring-loaded collapsible assembly mandrel with the collar pack position prior to the collaring.

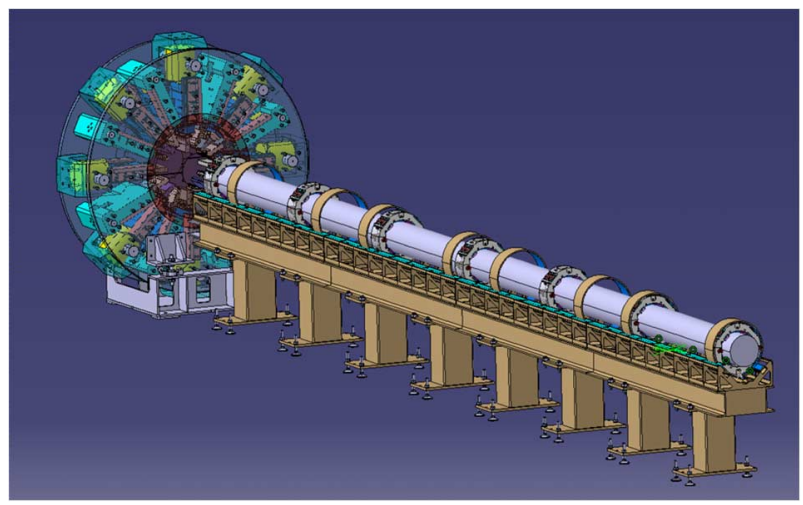

Fig. 13. Artistic view of the horizontal collaring press with a coil-support bench.

The winding topology of the coil allows a minimization of the integrated b6 component, however, the b10 is always negative and can, therefore, not be eliminated. If necessary, this integrated field error will have to be part-compensated for by the layout of the coil cross section.

\section{Horizontal COllaring PRess}

The collaring of the nearly 10 -m-long quadrupole magnets cannot be done with the standard presses used for the long dipole magnets. The reason is that the four-fold symmetry of the quadrupoles has to be guaranteed in order to avoid non-allowed multipole field error in the aperture field. Using a hydraulic press with anvils in horizontal direction would require a 10 -m-deep pit and a clearance under a 30-tone crane of more than 10 meters. There is no workshop at CERN that meets these requirements.
A novel self-locking collar pack design was therefore developed. These packs allow the assembly of the collars in horizontal direction, but require the above-mentioned, springloaded and collapsible assembly mandrel. The collars and assembly mandrel are shown in Fig. 12.

The novel collaring scheme needs to be qualified for the series production. This can be achieved with the aforementioned, instrumented collar pack. The hydraulic press needed for collaring will be procured from European industry. An artist's view of the press is shown in Fig. 13. The angular position of the collaring keys of the MQXC magnet is identical to the LHC main quadrupole and insertion magnets. If needed, the press can therefore be used also to refurbish these magnets.

\section{CONCLUSION AND OUTLOOK}

Although using available cable, and building on the past experience from the LHC magnet construction, the design, in-house production, and testing of the MQXC quadrupole magnets is not free of technical challenges. A particular challenge is to obtain the necessary pre-stress and field quality (coil size) in the magnet after cool-down and excitation using the new insulation scheme.

The MQXC magnet program foresees to construct at least two 2-m-long model magnets. Model 1 for the cold testing in a vertical cryostat, for field quality measurements, and training performance. Model 2 with full cold mass integration and cold testing in a horizontal cryostat. Model 2 will have extended instrumentation for heat transfer, quench propagation velocity measurements, and the study of the quench-back effect with differently sized protection resistors. If funding and resources permit, a third model construction could aim at the field quality required by the accelerator.

\section{REFERENCES}

[1] J. P. Koutchouk, L. Rossi, and E. Todesco, "A solution for phase-one upgrade of the LHC low-beta quadrupole based on Nb-Ti," CERN, LHC-Project-Report 1000, 2007.

[2] R. Ostojic, "LHC interaction region upgrade-Phase I," CERN, LHC Project Report 1094, 2008.

[3] F. Borgnolutti, P. Fessia, and E. Todesco, "Electromagnetic design of the $120 \mathrm{~mm}$ aperture quadrupole for the LHC phase one upgrade," CERN, SLHC Project Report 0001, 2009.

[4] M. La China and D. Tommasini, "Comparative study of heat transfer from $\mathrm{Nb}$-Ti and $\mathrm{Nb}_{3} \mathrm{Sn}$ coils to He II," Phys. Rev. ST Accelerators and Beams, vol. 11, 2008.

[5] P. Fessia, A. Gerardin, P. P. Granieri, M. Guinchard, S. Luzieux, S. Sgobba, and D. Tommasini, "Electrical and mechanical performance of an enhanced cable insulation scheme for the superconducting magnets of the LHC luminosity upgrade," IEEE Trans. Appl. Supercond., vol. 20, no. 3, 2010.

[6] R. Gupta et al., "Tuning shims for high field quality in superconducting magnets," IEEE Trans. Magn., vol. 32, no. 4, 1996.

[7] S. Russenschuck, Field Computation for Accelerator Magnets. Berlin: Wiley-VCH, 2010.

[8] F. Cerutti, M. Mereghetti, and E. Wildner, "Update of power deposition studies in the LHC upgrade phase I insertion region," CERN, EDMS Document No. 975134, 2008.

[9] R. Van Weelderen, "Update of the cryo-scheme," CERN, EDMS Document 1013620, 2009.

[10] R. Ostojic and T. Taylor, "Conceptual design of a $70 \mathrm{~mm}$ aperture quadrupole for the LHC insertions," IEEE Trans. on Appl. Supercond., vol. 3 , no. 1,1992 